\title{
Intracoronary Imaging and Plaque Vulnerability
}

\author{
Pál Maurovich-Horváth, Béla Merkely \\ Heart and Vascular Center, Faculty of Medicine, Semmelweis University, Budapest, Hungary
}

The concept of vulnerable atheromatous plaques and its characterization have been described in an attempt to elucidate the pathophysiological substrate of acute coronary syndromes. ${ }^{1,2}$ A vulnerable coronary plaque is defined as a plaque which is prone to rupture or erosion, triggering an acute coronary event. ${ }^{3,4}$ Following the description of the main features associated with coronary plaque vulnerability, this concept has been extended to carotid plaques, describing a condition, which is frequently associated with plaque embolization and stroke. ${ }^{5}$

However, not all vulnerable plaques result in an acute cardiovascular event. A significant number of unstable coronary plaques do not lead to acute coronary syndromes and their evolution can be regressed using statin therapy. ${ }^{6-8}$

In recent years it has been acknowledged that besides plaque characteristics, a multitude of factors are involved in the development of an acute coronary event or stroke, and the concept of plaque vulnerability was extended to the larger concept of patient vulnerability, describing a clinical situation associated with a generalized vulnerability of the patient following an exacerbated inflammation..$^{9-11}$ The interrelation between plaque vulnerability and patient vulnerability plays a significant role in determining the development of a coronary plaque into an acute cardiovascular event or towards regression and stabilization.

While patient vulnerability is characterized using sophisticated biomarkers associated with inflammation, features of plaque vulnerability have been studied using advanced imaging techniques.

The recent development of Cardiac Computed Tomography Angiography (CCTA) methods allows the noninvasive visualization of plaque features. Several plaque characteristics demonstrated good specificity and sensitivity for predicting an acute coronary event. ${ }^{12,13}$ A significant amount of low-density plaque, a high plaque burden, the presence of spotty calcifications, active remodeling and the napkin-ring sign inside the plaque have been identified as primary characteristics associated with plaque vulnerability. All these features are present to a larger extent in culprit lesions compared with non-culprit lesions of the coronary arteries, at the same time being associated with a higher rate of recurrent cardiac events and worse outcomes. ${ }^{14,15}$ Despite having the advantage of non-invasive visualisation of coronary plaques, CCTA is not able to provide relevant information on the thickness of the fibrous cap, a major feature of plaque vulnerability. Furthermore, it carries the risk of additional radiation to that delivered to the patient during a revascularization procedure.

A new noninvasive biomarker of plaque vulnerability is the shear stress, determined using CCTA data. ${ }^{16}$ Determination of the shear stress inside the coronary lumen, at the location of the plaque, is based on computational fluid dynamics. Studies assessing shear stress using this approach identified areas with low shear stress in the close vicinity of unstable coronary plaques. This implies that the alteration of intracoronary mechanical forces is involved in development of a vulnerable plaque. ${ }^{17,18} \mathrm{How}-$ ever, the procedure of shear stress determination is timeconsuming, therefore the technique cannot be used as a clinical decision tool in emergency conditions.

A study published in this issue of the journal by Benedek $\mathrm{T}$ et al. addresses the topic of plaque characterization using invasive intracoronary imaging. ${ }^{19}$ After reviewing and analyzing eleven studies published in the literature on the subject of vulnerable plaques characterization using OCT and IVUS, the most relevant markers associated 
with vulnerable plaquesm identifiable by invasive imaging, were described. The study proves that plaque burden, remodeling index, cross-sectional area, and the amount of necrotic core are the most relevant IVUS markers associated with vulnerable plaques, while the thickness of the fibrous cap, lipid arch, accumulation of macrophages and the presence of intracoronary thrombus are the most sensitive OCT markers of unstable plaques. ${ }^{19}$

At the same time, the study suggests that in patients with acute coronary syndromes, due to the particularities of the invasive approach, invasive imaging should be preferred rather than a noninvasive one, as it avoids unnecessary delays associated with CCTA before revascularization.19

The authors concluded that both these invasive techniques are complementary and can serve as useful clinical decision tools in ACS patients who are subject to a revascularization procedure.

Interestingly, the study addresses the topic of plaque vulnerability from a mixed approach, examining coronary plaques in culprit versus non-culprit lesions, in lipid-rich versus non-lipid rich plaques, in plaques with thin cap fibroatheroma versus those with thick cap fibroatheroma, and in ruptured versus non-ruptured plaques. This approach reflects the current advances in research dedicated to vulnerable plaque features, which indicated that not all vulnerable plaques have a TCFA and not all of them rupture. Histopathologic studies demonstrated that a significant percentage of lesions responsible for an ACS present plaque erosion with an intact fibrous cap, indicating that the classical concept of plaque rupture as the cause of thrombus formation and acute myocardial infarction is not valid in all cases. ${ }^{20,21}$

Compared to the many studies reporting CCTA-derived plaque features in different clinical settings, the number of studies included in this analysis is surprisingly small. This is probably explained by the low number of studies published in the literature, which included both OCT and IVUS assessment. However, the results of this systematic review undoubtedly indicate that in the era of noninvasive imaging, there is still a role for invasive assessment of coronary plaques using intracoronary imaging, especially in cardiac emergencies.

\section{REFERENCES}

1. Finn AV, Nakano M, Narula J, Kolodgie FD, Virmani R. Concept of vulnerable/unstable plaque. Arterioscler Thromb Vasc Biol. 2010;30:1282-1292. doi: 10.1161/ATVBAHA.108.179739.

2. Stone GW1, Maehara A, Lansky AJ, et al. A prospective natural-history study of coronary atherosclerosis. N Engl J Med. 2011;364:226-235. doi: 10.1056/NEJMoa1002358.
3. Bentzon JF, Otsuka F, Virmani R, Falk E. Mechanisms of plaque formation and rupture. Circ Res. 2014;114:1852-1866. doi: 10.1161/CIRCRESAHA.114.302721.

4. Tian J, Ren X, Vergallo R, et al. Distinct morphological features of ruptured culprit plaque for acute coronary events compared to those with silent rupture and thin-cap fibroatheroma: a combined optical coherence tomography and intravascular ultrasound study. J Am Coll Cardiol. 2014;63:2209-2216. http://www.ajronline.org/doi/abs/10.2214/AJR.14.13760.

5. Nighoghossian N1, Derex L, Douek P. The vulnerable carotid artery plaque: current imaging methods and new perspectives. Stroke. 2005;36:2764-2772. doi: 10.1161/01. STR.0000190895.51934.43.

6. Dave T, Ezhilan J, Vasnawala H, Somani V. Plaque regression and plaque stabilisation in cardiovascular diseases. Indian J Endocrinol Metab. 2013;17:983-989. doi: 10.4103/22308210.122604 .

7. Bittencourt MS, Cerci RJ. Statin effects on atherosclerotic plaques: regression or healing? BMC Medicine. 2015;13:260. doi:10.1186/s12916-015-0499-9.

8. Yia-Hertualla S, Fog Bentzon J, Daemen M, et al. Stabilization of atherosclerotic plaques: an update. Eur Heart J. 2013; 34:3251-3258. doi: 10.1093/eurheart/eht301.

9. Naghavi M, Libby P, Falk E, et al. From vulnerable plaque to vulnerable patient: a call for new definitions and risk assessment strategies: Part I. Circulation. 2003;108:16641672. doi: 10.1161/01.CIR.0000087480.94275.97.

10. Dalager MG, Bøttcher M, Thygesen J, Andersen G, Bøtker HE. Different Plaque Composition and Progression in Patients with Stable and Unstable Coronary Syndromes Evaluated by Cardiac CT. BioMed Research International. 2015;2015:401357. doi:10.1155/2015/401357.

11. Waxman S, Ishibashi F, Muller JE. Detection and Treatment of Vulnerable Plaques and Vulnerable Patients. Novel Approaches to Prevention of Coronary Events. Circulation. 2006;114:23902411. doi: 10.1161/CIRCULATIONAHA.105.540013.

12. Thomsen C, Abdulla J. Characteristics of high-risk coronary plaques identified by computed tomographic angiography and associated prognosis: a systematic review and meta-analysis. Eur Heart J Cardiovasc Imaging. 2016;17:120-129. doi: 10.1093/ ehjci/jev325.

13. Motoyama S, Masayoshi S, Harigaya H, et al. Computed tomographic angiography characteristics of atherosclerotic plaques subsequently resulting in acute coronary syndrome. J Am Coll Cardiol. 2009;54:49-57. doi: 10.1016/j. jacc.2009.02.068.

14. Nakazato R, Otake H. Konishi A, et al. Atherosclerotic plaque characterizationby CT angiography for identification of highrisk coronary artery lesions: a comparison to optical coherence tomography. Eur Heart J Cardiovasc Imaging. 2015;16:373379. doi: 10.1093/ehjci/jeu188.

15. Benedek T, Gyöngyösi M, Benedek I. Multislice Computed Tomographic Coronary Angiography for Quantitative Assessment of Culprit Lesions in Acute Coronary Syndromes. Can J Cardiol. 2013;29:364-371. doi: 10.1016/j.cjca.2012.11.004.

16. Choi G, Lee JM, Kim HJ, et al. Coronary Artery Axial Plaque Stress and its Relationship With Lesion Geometry: Application of Computational Fluid Dynamics to Coronary CT Angiography. JACC Cardiovasc Imaging. 2015;8:1156-1166. doi: 10.1016/j. jcmg.2015.04.024. 
17. Park JB, Choi G, Chun EJ, et al. Computational fluid dynamic measures of wall shear stress are related to coronary lesion characteristics. Heart. 2016;102:1655-1661. doi: 10.1136/ heartjnl-2016-309299.

18. Wentzel JJ, Chatzizisis YS, Gijsen FJ, Giannoglou GD, Feldman $\mathrm{CL}$, Stone PH. Endothelial shear stress in the evolution of coronary atherosclerotic plaque and vascular remodelling: current understanding and remaining questions. Cardiovasc Res. 2012;96:234-243. doi: 10.1093/cvr/cvs217.

19. Benedek T, Mester A, Benedek A, Rat N, Opincariu D, Chițu M. Assessment of Coronary Plaque Vulnerability in Acute Coronary Syndromes using Optical Coherence Tomography or Intravascular Ultrasound. A systematic Review. Journal of Cardiovascular Emergencies. 2016;2(4):173-184. doi: 10.1515/ jce-2016-0028.

20. White SJ, Newby AC, Johnson TW. Endothelial erosion of plaques as a substrate for coronary thrombosis. Thromb Haemost. 2016;115:509-519. doi: 10.1160/TH15-09-0765.

21. Nasu K, Tsuchikane E, Katoh O, et al. Accuracy of in vivo coronary plaque morphology assessment: a validation study of in vivo virtual histology compared with in vitro histopathology. J Am Coll Cardiol. 2006;47:2405-2412. doi: 10.1016/j.jacc.2006.02.044. 